\title{
Groin Pain in Athletes
}

\author{
Leistenschmerz beim Sportler
}

Authors

Affiliations
M.-A. Weber ${ }^{1}$, C. Rehnitz ${ }^{1}$, H. Ott ${ }^{2,3}$, N. Streich ${ }^{2,4}$

Affiliation addresses are listed at the end of the article.

Key words
bones
muscular
pelvis
MR imaging
ultrasound
trauma

eingereicht 6.11 .2012

akzeptiert 3.2.2013

Bibliography

Dol http://dx.doi.org/

10.1055/s-0033-1335304

Published online: 26.7.2013

Fortschr Röntgenstr 2013; 185 :

1139-1148 @ Georg Thieme

Verlag KG Stuttgart · New York .

ISSN 1438-9029

\section{Correspondence}

Herr Prof. Marc-André Weber Department of Diagnostic and Interventional Radiology, University Hospital Heidelberg Im Neuenheimer Feld 110 69120 Heidelberg Tel.: ++49/6221/5637434 Fax: ++49/6221/5626640 MarcAndre.Weber@med. uni-heidelberg.de

\section{Abstract \\ $\nabla$}

Groin pain in athletes is one of the most difficult to treat clinical entities in sports medicine. The reasons are the amount of differential diagnoses, complexity of pathophysiologic causes and the long time of limited participation in sport. In order to maximize efficient treatment, thorough diagnostics and a clear therapeutic regimen are crucial. To succeed with this issue, a close cooperation between physicians and radiologists is mandatory. MRI is gold standard in the diagnostic work-up of the principal differential diagnoses, such as muscle tears, avulsion injuries, stress fractures, tears of acetabular labrum, and osteitis pubis. The article gives a comprehensive overview of the special anatomy and biomechanics of the pubic region and of typical MRI findings in athletes with groin pain. The use of dedicated imaging protocols is also discussed.

Key Points:

- Groin pain in competitive sports is frequent (5-18\% incidence among professional soccer and tennis players).

- The differential diagnoses are numerous and comprise among others extraarticular (e.g. muscle and tendon injuries, stress fractures), intraarticular causes (e.g. femoroacetabular impingement, labral lesions), and osteitis pubis.

- Sports hernia is a clinical diagnosis, the radiologist should exclude or define other causes such as osteitis pubis.

- A labral tear as cause of groin pain can only be reliably diagnosed using direct MR arthrography.

Citation Format:

- Weber MA, Rehnitz C, Ott H etal. Groin Pain in Athletes. Fortschr Röntgenstr 2013; 185: 1139-1148

\section{Zusammenfassung \\ $\nabla$}

Der Leistenschmerz beim Profisport ist eines der am schwierigsten zu behandelnden Krankheitsbilder in der Sportmedizin. Grund hierfür ist die Fülle von Differenzialdiagnosen, die Komplexität der pathophysiologischen Einflussfaktoren und die mitunter lange Ausfallzeit. Um eine maximal effiziente Therapie zu gewährleisten, sind eine differenzierte Diagnostik und ein klares Therapiekonzept entscheidend. Dies gelingt nur, wenn eine enge $\mathrm{Zu}$ sammenarbeit zwischen betreuenden Ärzten und Radiologen stattfindet. Die MRT ist der Goldstandard zur Aufarbeitung der wichtigsten Differenzialdiagnosen wie Muskelverletzungen, apophysäre Verletzungen und Stressfrakturen, Labrumpathologien des Hüftgelenks und die Osteitis pubis. Der Artikel gibt einen umfassenden Überblick über die spezielle Anatomie und die Biomechanik der Schamgegend und typische MRT-Befunde bei Athleten mit Leistenschmerzen. Die Verwendung eines dezidierten Bildgebungsprotokolls wird ebenso diskutiert.

\section{Introduction \\ $\nabla$}

Groin pain in competitive sports is a frequent and complex ailment accounting for $2-5 \%$ of all sports-related pain development, with an incidence of $5-18 \%$ among professional soccer and tennis players [1-5]. Chronic groin pain frequently leads to extended time lost to injury, despite all therapy [6]. Consequently, in recent years increasing value has been placed on prophylaxis and early detection. Important in this regard is a precise diagnosis of the causes of the complaint; for establishing a correct diagnosis the close collaboration between the attending (team) physician and radiologist is indispensable. Especially in professional sports, which enjoy extensive media coverage, there is great pressure on physicians 
and therapists, since the athlete's downtime should be minimized, and the prognosis generally cannot be precisely provided to the trainer and athlete. Further, groin pain is likewise an increasing problem in recreational sports, with an incidence of sport-related injuries of 3.1-5.6\% [7]. One need only consider the many middle-aged casual athletes, mainly with sedentary occupations, "weekend warriors" who vigorously pursue sports during their free time. The following provides an overview of the epidemiology of groin pain among competitive athletes, identifies frequent differential diagnoses, and describes diagnostics and therapy with a focus on ultrasound and MRI procedures. In particular, this article will discuss muscle injuries in the groin region, apophyseal injuries and stress fractures as well as labral pathology of the hip joint and osteitis pubis.

\section{Epidemiology}

$\nabla$

In competitive sports, groin pain represents one of the most frequent complaints and is observed among professional athletes, primarily soccer, hockey or rugby players [8-10]. In addition, groin pain occurs in track and field athletics (e. g. hurdling), volleyball or ski racing [11]. Common to all these sports are recurrent, abruptly flexing and rotational movements in the hip joint and groin with rapid changes of direction and high torqueing [5]. As a consequence, the fascia as well as musculoskeletal structures of the thigh and caudal abdominal musculature are strained. Whereas acute groin pain can be treated with conservative remedies within 4 to 6 weeks, symptoms of chronic groin pain are persistent and frequently result in considerable time lost due to injury [9]. With respect to causes of downtime, inguinal pain is in third place, behind fractures and injuries to the anterior and posterior cruciate ligament [12].

The frequency of groin complaints is steadily increasing among soccer players. As early as 1966, Cabot noted that in the previous 30 years, $0.5 \%$ of Spanish footballers complained of groin pain; in 1980, Renström stated that 5 $13 \%$ of players presented with groin-related pain within an observation period of $1-2$ years [13]. In the meantime, approximately $60 \%$ of soccer players report acute or chronic groin pain during their playing years, and every tenth injury-related absence from play is related to groin pain or injury $[1,14]$. Causes mentioned include increasing playing and training demands, increased motional speed and the development of footwear with increased traction resulting from changes to the cleats. In a prospective study of $23 \mathrm{Eu}-$ ropean top member teams of the UEFA (Union des Associations Européennes de Football), between 2001 and 2007 an average of $12-16 \%$ of all injuries per team were related to groin or hip injuries per season [10]. These numbers concur with those of the German soccer league team, TSG 1899 Hoffenheim, with which we are affiliated.

\section{Differential Diagnoses}

$\nabla$

The groin and hip region is made up of a number of different structures that interact: pubic bones, pubic symphysis, musculature, tendons, aponeuroses, ligaments, inguinal canal, hip joint. Consequently there is frequently reference pain or a pain pattern that cannot be uniquely assigned to a single structure $[1,3]$. In addition, there are a multitude of various nerves responsible for providing sensation and which support movement. Compression of these nerves (entrapment syndrome), such as a result of laxness of muscle fascia, can likewise lead to significant stress-related pain $[1,5]$. Therefore a knowledge of the complex anatomy of the groin and pelvic region, interaction of the affected structures and a detailed clinical investigation, as well as dedicated image-based diagnostics are prerequisites for a definitive diagnosis with respect to the numerous differential diagnostic-related causes $[5,6]$. Further, close interdisciplinary collaboration among orthopedists and trauma surgeons with focus on sports medicine together with radiologists is required to establish appropriate imaging protocols. - Table 1 provides a summary of possible differential diagnoses, whereby a distinction must be made between extraarticular causes (e.g. muscle and tendon injuries) and intraarticular sources (e.g. femoroacetabular impingement, labral lesions) $[1,5,6,15-18]$. The following sections present the most frequent causes in detail.

\section{Muscular Injuries in the Groin Region and Apophyseal Injuries}

Muscular injuries of the adductors, the iliopsoas muscle and abdominal musculature are the most frequent sources for the occurrence of acute groin pain; among 23 UEFA top teams, adductor injuries $(\mathrm{n}=399 / 628)$ were most frequent, followed by injuries to the iliopsoas muscle $(n=52 / 628)$ [10]. Depending on the age of the athlete, muscular weak points include unfused apophysis among the young, degenerative tendons among more mature and older athletes. Among most competitive athletes between the ages of 20 and 30, the myotendinous junction is the predilection site for muscle tears $[15,19]$. When the immature skeletal struc-

Table 1 Frequent causes of groin pain in competitive athletics, modified according to [15]

\begin{tabular}{|c|c|}
\hline \multicolumn{2}{|l|}{ acute onset } \\
\hline & $\begin{array}{l}\text { muscle injury (strain, muscle fiber rupture, } \\
\text { fascicle rupture etc.) }\end{array}$ \\
\hline & avulsion and apophyseal injury \\
\hline & fracture \\
\hline \multicolumn{2}{|l|}{ gradual onset } \\
\hline & adductor inflammation, insertional tendinosis \\
\hline & osteitis pubis, symphysitis \\
\hline & weak groin, inguinal hernia \\
\hline & $\begin{array}{l}\text { pathology of the hip joint (labral lesions, } \\
\text { femoroacetabular impingement (FAI)) }\end{array}$ \\
\hline & snapping hip (coxa saltans) \\
\hline \multicolumn{2}{|l|}{ spinal column disorders } \\
\hline & disc prolapse with nerve root inflammation \\
\hline & blockage in the lumbar spine region \\
\hline & spondylolisthesis \\
\hline \multicolumn{2}{|l|}{$\begin{array}{l}\text { ilioinguinal nerve } \\
\text { entrapment syndrome }\end{array}$} \\
\hline \multicolumn{2}{|l|}{$\begin{array}{l}\text { functional impairment } \\
\text { of the pelvis }\end{array}$} \\
\hline & blockage of the iliosacral joints \\
\hline & pelvic torsion \\
\hline $\begin{array}{l}\text { imbalance in the muscle } \\
\text { strings }\end{array}$ & \\
\hline
\end{tabular}



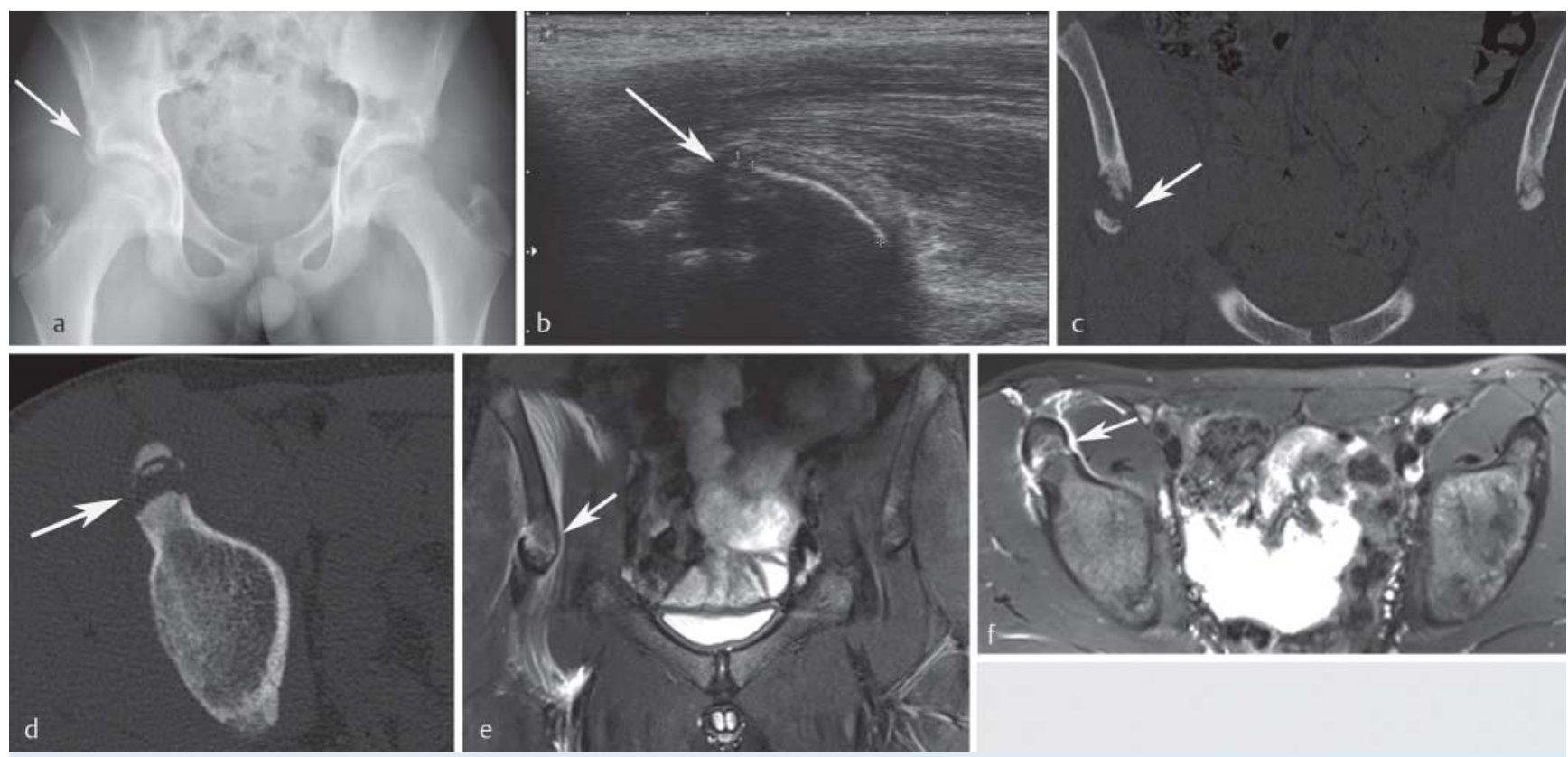

Fig. 1 Apophyseal muscle detachment. 13-year-old youth soccer player with apophyseal detachment of the rectus femoris muscle on the right anterior inferior iliac spine (arrows). Pelvic overview image a, ultrasound $\mathbf{b}$,

$\mathrm{CT}$ in coronal $\mathbf{c}$ and axial reformation d, 3-Tesla MRI: coronal STIR sequence $\mathbf{e}$, axial, T2-weighted fat-suppressed sequence $\mathbf{f}$.

ture is overstressed, injuries to the apophysis can occur at all large muscle attachments [15] ( $\bullet$ Fig. 1). In mature skeletal structures, avulsions occur practically only among highperformance athletes.

Whereas the ischiocrural muscles (hamstrings) are most frequently affected, and such injuries generally occur during running, in the case of adductors, injuries arise as a result of abrupt rotational motions or trauma during abduction or adduction rotation [19]. An early diagnosis established using imaging is critical for proper treatment since prompt and intensive treatment as well as complete healing of these injuries is crucial in avoiding development into a potentially chronic inflammation of the muscle insertion. Ultrasonography is the initial imaging modality in the case of simple superficial injuries. Advantages include high local resolution, rapid access, dynamic examination in real time and costeffectiveness. The disadvantages, however, include dependence on the examiner's experience, poor reproducibility, less longitudinal coverage than MRI, limitation with respect to deep lesions or those close to the pubic bone [6]. On the other hand, MRI is the procedure of choice for complex injuries and for prognostication, in particular with respect to high-performance sports [6], since these injuries require exact assessment of the length of the muscle tear, and is reproducible when evaluating progress. A muscular edema verified by MRI can last longer than the actual pain, and can indicate increased vulnerability of the muscle [20]. In addition to the presence of a hematoma, the longitudinal extension of a muscle tear is a decisive predictor of lost playing time $(r=0.58$; $\mathrm{p}<0.0001$ ) [21]; this downtime can be underestimated by ultrasonography [22]. The assessing radiologist should indicate the degree of the muscle tear, as this is important for the type of therapy to be selected as well as useful for estimating the downtime and risk of recurrence. In Germanspeaking areas, the Müller-Wohlfahrt classification of Grades
Table 2: Overview of common graduations of muscle injury.

\begin{tabular}{|c|c|}
\hline \multirow[t]{2}{*}{ Müller-Wohlfahrt 2010 [23]: } & Peetrons 2002 [24]: Rybak 2003 [19]: \\
\hline & (ultrasound-based) \\
\hline grade I: muscle hardening & \multirow[t]{2}{*}{ grade 0 : no changes visible in imaging } \\
\hline $\begin{array}{l}\text { grade II: so-called "muscle } \\
\text { strain" (neuromuscular strain) }\end{array}$ & \\
\hline $\begin{array}{l}\text { grade Illa: muscle fiber rupture } \\
\text { ( } \leq 5 \mathrm{~mm} \text { transverse extension) }\end{array}$ & $\begin{array}{l}\text { grade 1: muscular edema. Discontinuity } \\
<5 \% \text { of muscle fiber. Perifascial fluid col- } \\
\text { lection. Possibly minimal hematoma, no } \\
\text { function deficit. }\end{array}$ \\
\hline $\begin{array}{l}\text { grade IIIb: muscle bundle tear } \\
\text { ( } \geq 5 \mathrm{~mm} \text { transverse extension; } \\
\text { exceptional form: intramuscular } \\
\text { tendon rupture) }\end{array}$ & $\begin{array}{l}\text { grade } 2 \text { : partial rupture }>5 \% \text { discontinu- } \\
\text { ity. Hematoma, functional limitation. } \\
\text { Indicate proportional transverse and } \\
\text { longitudinal extension. }\end{array}$ \\
\hline $\begin{array}{l}\text { grade IV: muscle tear/tendinous } \\
\text { muscle detachment }\end{array}$ & $\begin{array}{l}\text { grade 3: complete rupture. Muscle gap, } \\
\text { avulsed muscle and tendon origins. } \\
\text { Almost always hematoma. Significant } \\
\text { functional limitations, OP if needed. }\end{array}$ \\
\hline
\end{tabular}

I-IV is common [23]; internationally, classification uses Grade 1 (feathery edema), Grade 2 (partial tear) and Grade 3 (complete tear with a fluid-filled gap) $[19,24,25]$ ( $\bullet$ Table 2 , - Fig. 2; Overview and consensus recommendation [26]).

\section{Stress fractures}

Stress responses should additionally be considered as a cause of groin pain; in athletes these occur mainly in the lower pubic branch, the pubic symphysis and proximal femur $[5,6,17,18]$. Stress fractures are generally observed in female runners; to avoid them, the training distance should not be increased by more than $10 \%$ per week [15]. It is important to consider that the conventional X-ray image frequently yields negative results; MRI is the most sensitive examination method $[5,6]$ ( $\odot$ Fig. 3 ). A radionuclide scan to 

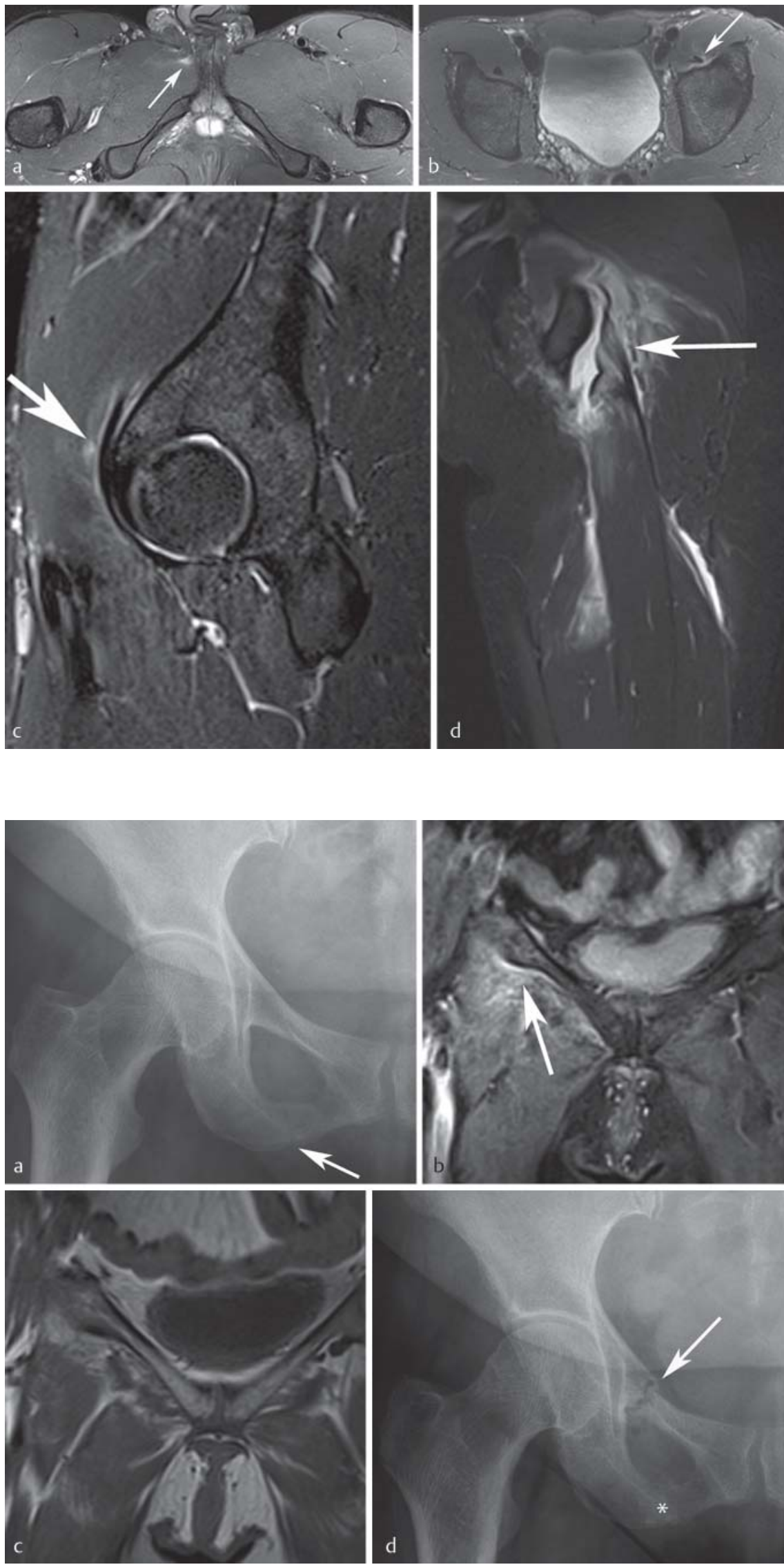

Fig. 2 Overview of muscle tears with varying degrees of severity in the vicinity of the pelvis. a The axial fat-suppressed T2-weighting shows a grade 1 tear according to [19] of the right adductor magnus muscle (arrow) in a 20-year-old professional soccer player. b, c Grade 1 tear according to [19] of the left iliopsoas muscle on the myotendinous junction (arrow) in a 30-year-old professional soccer player (b, axial fat-suppressed T2-weighted; c, sagittal STIR sequence). $\mathbf{d}$ Grade 3 muscle injury according to [19] after a split during a dance performance of a 15-year-old girl with detachment of the biceps femoris muscle on the ischial tuberosity. The arrow on the coronal STIR sequence points to the detached tendon end and the hematoma.

Fig. 3 Stress fractures. The conventional X-ray image $\mathbf{a}$ exhibits no stress fracture on the upper pubic branch, but rather a fracture line through the lower pubic branch (arrow). The 3-Tesla MRI (b, coronal STIR; c, coronal T1-weighting) can verify the periostal accentuated osseous edema as an indication of the stress reaction of the right superior pubic ramus, and the edema of the surrounding muscles and soft tissues (arrow) (grade 3 stress fracture according to Kiuru [6]). Despite an interruption of sports activity, two months later the woman experienced a stress fracture of the upper pubic branch (arrow in $\mathbf{d}$ ); there is callus formation around the stress fracture of the lower pubic branch (asterisk in d). 
demonstrate a stress fracture is obsolete $[5,6]$ and is only used in individual cases when MRI is contraindicated and CT findings are inconclusive.

\section{Osteitis Pubis or Symphysitis}

Both of these diagnoses concern one of the most frequent causes of chronic groin pain among soccer and rugby players, American football players as well as runners and swimmers (primarily among competitive athletes performing kicking motions, rapid acceleration and deceleration movements as well as abrupt changes in direction) [27]; in our experience, hardly any professional soccer players exhibit a completely unremarkable symphysis in dedicated imaging procedures. The cause is chronic insertional tendinosis of the adductors resulting from repetitive trauma reinforced by an imbalance between the adductors and the rectus abdominis muscle. As this progresses, the pubic symphysis becomes more instable with consecutive stress reactions of this joint and adjacent pubic bone branches $[5,6]$. The result is a vicious circle of symphysitis, joint instability and muscular imbalance of the originating muscles. Medial pelvic pain, particularly tenderness of the pubic symphysis and pubic bones are usually present as well as generally positive adductor signs; the pain can, however, project into the abdomen, either perineally or in the direction of the scrotum [27]. The related therapy is difficult, frequently long-term, and re- quires a complete spectrum ranging from physiotherapy, pain medication, local injections (corticoides [28], local anesthesia [28], autologous conditioned plasma [29]), through hyperbaric oxygen therapy in a pressure chamber [30], and potentially, denervation or fusion operations [6, 27]. An extended absence from sports activity is generally unavoidable. Downtime is frequently 3 months or longer $[5,6]$.

Familiarity with the following special anatomical characteristics is crucial for understanding the origin of osteitis pubis. The two pubic bones are separated by hyaline cartilage and a central fibrocartilaginous pubic disc, which in adult physiology exhibits a fluid-filled gap, the symphyseal cavity $[2,31]$. In the region of the pubic symphysis are important muscle groups such as the adductors (especially adductor longus) and the rectus abdominis muscles as well as elements of the inguinal canal that are bound to the pubic symphysis ( $\bullet$ Fig. 4a-c) $[17,18,32]$. The rectus abdominis muscle stabilizes the distal abdominal wall, and its insertions join with the sources of the adductor longus muscle ventrally and on the anterior aspect of the pubic symphysis $[33,34]$. These connective tissues and insertions form the prepubic aponeurotic complex [3], called also the rectus abdominis-adductor aponeurosis and inserts on the pubic tubercle $[5,17]$. Likewise, the inguinal ligament, an important element of the inguinal canal, moves in that direction, and these structures are associated with the periosteum
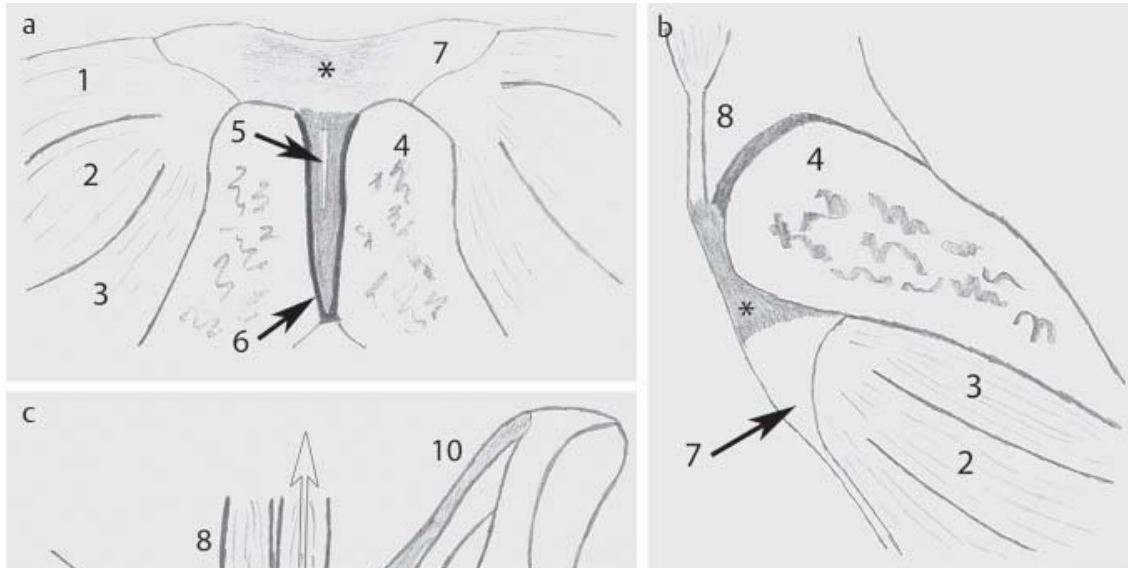

Fig. 4 Schematic illustrations of the anatomy of the symphysis region. a Axial sectional view through the symphysis and $\mathbf{b}$ sagittal sectional view $1-2 \mathrm{~cm}$ lateral to the symphysis center modified according to [3]. $\mathbf{c}$ The drawing illustrates the narrow position of the outer inguinal ring lateral to the rectus abdominis-adductor aponeurosis. The rectus and adductor longus muscles function antagonistically on the symphysis during rotation and extension; the open arrows illustrate the force vectors [5]. Targeted MRI orientation in the case of osteitis
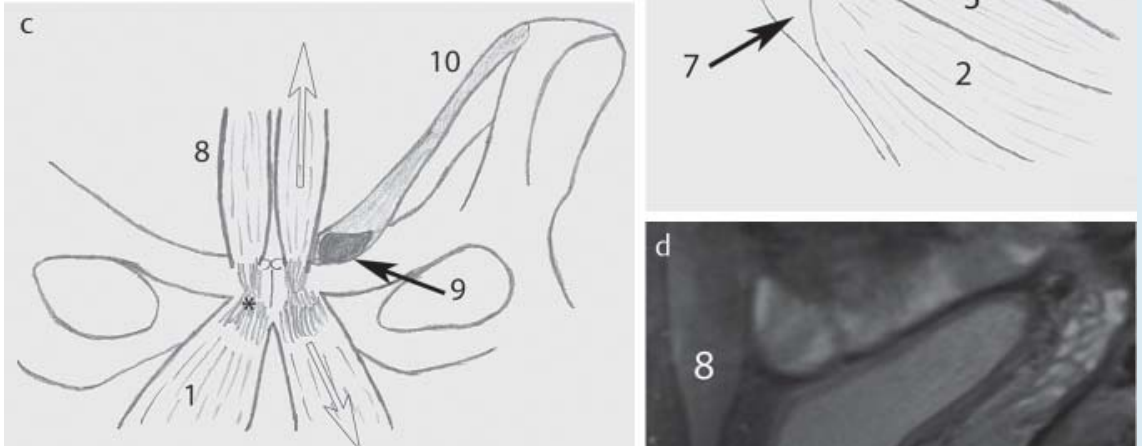
pubis. Methods of choice are a sagittal, fat-suppressed T2-weighting or proton density (PD)weighting to demonstrate the rectus abdominisadductor aponeurosis $\mathbf{d}$ and a T2-weighted sequence parallel to the arcuate line of the ilium, i. e. axial oblique e. Asterisks: rectus abdominis-muscle adductor aponeurosis, 1: adductor longus muscle, 2: adductor brevis muscle, 3: obturator externus muscle, 4: pubic bone, 5: symphyseal cavity in the interpubic disc, 6: hyaline cartilage, 7: adductor longus muscle insertion, 8: rectus abdominis muscle, 9: outer inguinal ring, 10: inguinal ligament, 11: obturator internus muscle.
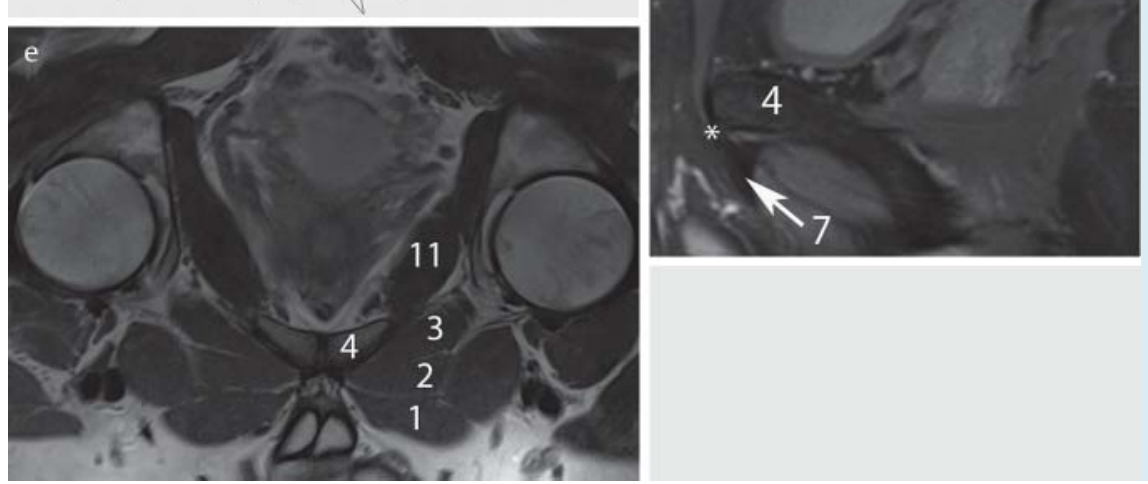
of the pubic bone, the ligaments and disc of the pubic symphysis ( $\bullet$ Fig. 4a-c) $[3,5,6,33,34]$. The outer inguinal ring lies less than $5 \mathrm{~mm}$ laterally to this aponeurosis [5, 32]. The anatomical features imply that injuries to the adductor longus muscle and rectus abdominis muscle involving the prepubic aponeurosis can affect the inguinal canal $[5,17]$. Detachment of the prepubic aponeurotic complex, either partial or complete, is an injury frequently designated as sportsman's hernia $[3,35]$ - probably because of the accompanying involvement of the inguinal canal [3]. However, the radiologist should avoid this term in favor of the more proper osteitis pubis [3]. Imbalance between abdominal and adductor muscles interfere with the force equilibrium on the pubic symphysis [6]. An injury to one of these components thus creates instability of the symphyseal region $[3,17]$. In addition, injuries to the adductor insert regularly lead to involvement of the insertion of the rectus abdominis muscle at the pubic symphysis $[5,18]$.

An important element in the MR diagnostic workup of suspected osteitis pubis is the representation of the pubic symphysis by means of an optimized sequence protocol ( $\bullet$ Table 3 ) using axially oblique orientation parallel to the arcuate line of the ilium [5] as well as sequences with a large field of view combined with high-resolution sequences ( $\bullet$ Fig. $\mathbf{4 d}$, e). Coronal sequences can be used to optimally assess the rectus abdominis muscle insertion and originating point of the adductor; sagittal proton density or T2-weighted fat-saturated sequences above the symphysis best represent the rectus abdominis-adductor aponeurosis with its periosteal attachments to the anterior and anterior-inferior pubic bone ( $\bullet$ Fig. 4 d) $[3,5,17]$. Im- age findings of osteitis pubis in acute cases reveal juxta-articulate bone marrow edema in the adjacent pubic bone (৫ Fig. 5), an irregular symphyseal cavity and frequently accompanying edema in all or individual adductor brevis, adductor longus and rectus abdominis muscles [6, 17]. As mentioned above, a lesion of these stabilizing muscles initially results in destabilization of the symphysis [5], thus setting the stage for osteitis pubis. Lesions of the rectus abdominis-adductor aponeurosis can range from minimal detachment to complete ruptures with retraction of the

Table 3 MRI protocol for diagnosing symphyseal / groin pain in competitive athletics

\begin{tabular}{llll} 
sequence & orientation & FOV $\mathbf{( c m )}$ & $\begin{array}{l}\text { slice thickness } \\
\text { [mm] }\end{array}$ \\
\hline STIR & coronal & $28-38$ & $3-4$ \\
\hline T1w & coronal & $28-38$ & $3-4$ \\
\hline T2w fat-suppressed & axial & $28-36$ & $3-4$ \\
\hline T2w & axial oblique & 20 & $2-3$ \\
\hline PD fat-suppressed & sagittal & $20-24$ & $2-3$ \\
\hline T2w fat-suppressed & axial oblique & 20 & $2-3$ \\
\hline T1w & axial oblique & 20 & $2-3$ \\
\hline Optional & & & \\
\hline T2w fat-suppressed & sagittal & $20-24$ & $2-3$ \\
\hline T1w after CM & axial oblique & 20 & $2-3$
\end{tabular}

FOV: Field-of-view, STIR: Short tau inversion recovery, T1w: T1-weighted sequence, T2w: T2-weighted sequence, PD: Proton density. Axial oblique: oblique layer parallel to the arcuate line of the ilium. A supplementary injection of contrast medium (CM) is necessary only in cases of suspected septic arthritis and is superfluous in the standard diagnosis of osteitis pubis. MR arthrography of the hip should be performed if a labral lesion is suspected.
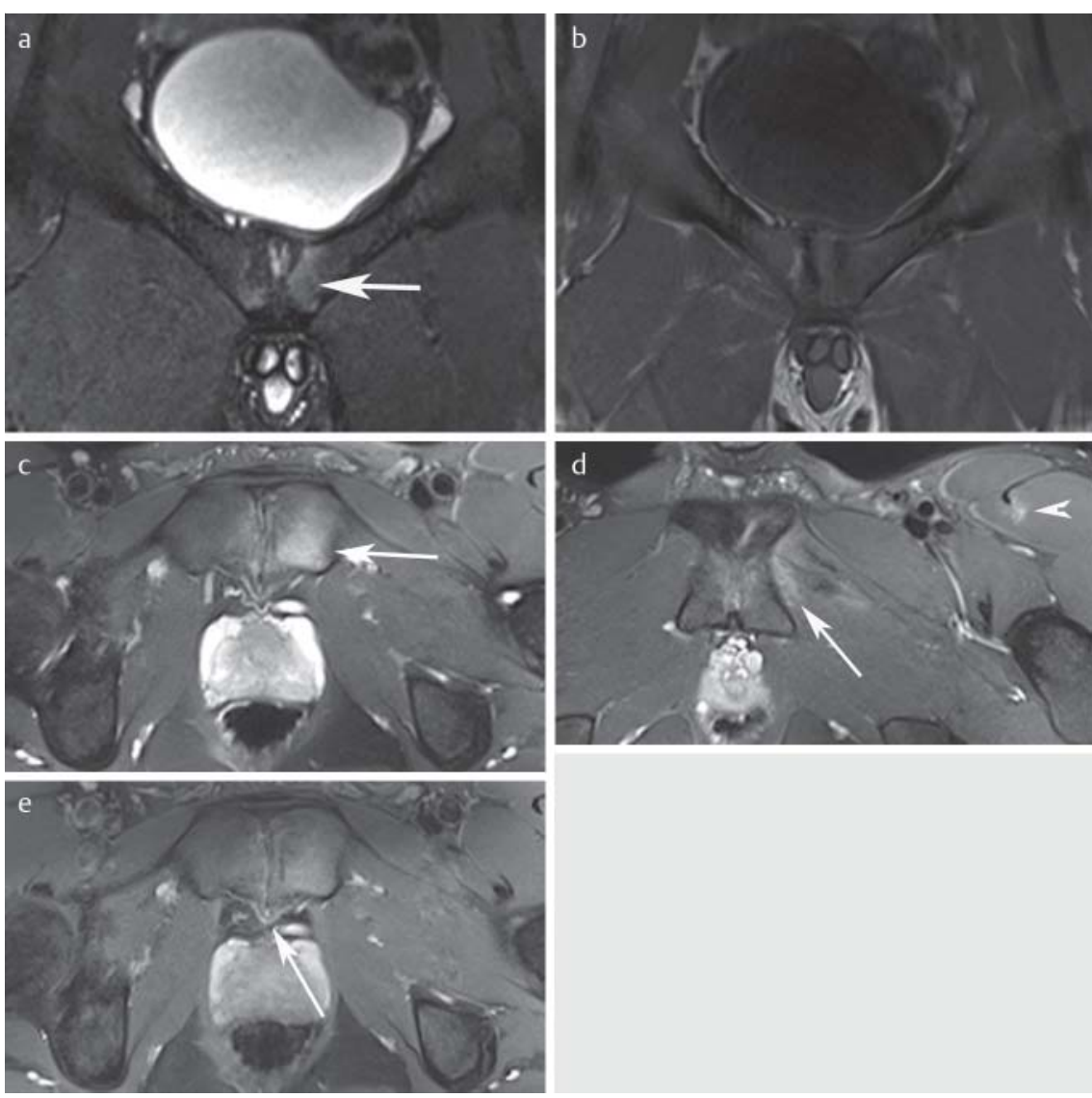

Fig. 5 Acute osteitis pubis. 17-year-old youth soccer player with acute osteitis pubis and bilateral subchondral, mainly left-sided parasymphyseal bone marrow edema (arrow), coronal STIR a and coronal T1-weighting $\mathbf{b}$. The maximum or side of the edema frequently correlates to the pain site. c, d Axial, fat-suppressed T2-weighted 3-Tesla MRI of a 20-year-old professional soccer player. Left-side parasymphyseal bone marrow edema (arrow) and edema in the left adductor brevis muscle insertion (arrow), both typical in acute cases. In addition, there is a grade 1 muscle tear according to [19] in the rectus femoris muscle (tip of arrow). A supplementary injection of contrast medium $\mathbf{e}$ is necessary only in cases of suspected septic arthritis and provided no additional utility in this case. Further, a posterior herniation of the symphyseal disc (arrow) is present, frequently encountered in competitive athletes, and which can be asymptomatic [6]. It is unclear whether this herniation predisposes to osteitis pubis [2]. 

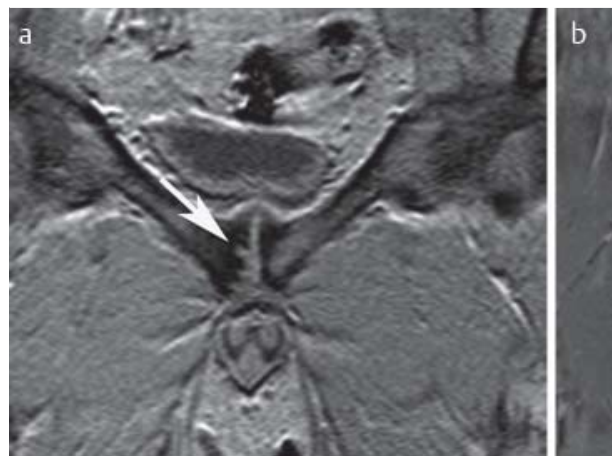

Fig. 6 Chronic osteitis pubis. 56-year-old male with chronic osteitis pubis. a The coronal T1-weighting demonstrates an irregular margin of the symphyseal cavity with erosions (arrow). b Active chronic osteitis pubis in a 30 year-old professional soccer player. The coronal STIR sequence shows left parasymphyseal bone marrow edema (asterisk). This bone marrow edema is an indicator of active involvement. The protrusion of the cranial symphysis (arrow) by osteophytes and hypertrophy of the capsule and superior pubic ligament as well as the irregular joint margin with subchondral cysts indicate a chronic process. insertions of the adductor longus and rectus abdominis muscles from the pubic symphysis and pubic tubercle. Milder lesions (micro-ruptures on the adductor origin) can manifest themselves as secondary cleft formation (primary cleft = symphyseal cavity) which can be delineated on coronal MRI images as T2w-hyperintense on the inferolateral edge of the pubic symphysis [2, 5, 31]. Some patients present with only isolated lesions of the adductor compartment, which in the MRI are displayed as minor injuries to the myotendinous junction ranging from a feathery $\mathrm{T} 2 \mathrm{w}$-hyperintense edema through chronic tendinosis of the adductor junction with enlarged hypointense tendon $[3,5]$. In the MRI, myotendinous injuries and muscle tears in the adductor compartment should be graded like other muscle tears (see above) [17, 32].

Chronic progression with symptoms lasting more than 6 months, for example in the case of chronic instability of the pubic symphysis resulting from repetitive microtrauma, exhibit typical indicators of arthrosis such as joint space narrowing, subchondral sclerosis and cysts as well as productive changes such as bony spurs and enthesiopathy of the adductors [6,32], whereas active chronic pubic osteitis exhibits appositional bone marrow edema ( $\bullet$ Fig. 6 ). On the pubic symphysis this process is frequently bilateral, although asymmetrical, and the side with the greatest MRI change is generally the side with the most pain $[2,17]$.

The differential diagnosis must take into account that ruptures of the hip flexors such as the sartorius, iliacus and iliopsoas muscles can clinically mimic an injury to the rectus abdominis-adductor aponeurosis complex [5, 6]. In this instance, MRI permits clear differentiation and reliably identifies accompanying pathologies. A radionuclide scan to demonstrate an osteitis pubis is obsolete [6] and is only used in individual cases to assess activity when MRI is contraindicated.

\section{Genuine Hernia, Sports Hernia and Weak Groin}

During diagnostic workup of groin pain, additional extraarticular pathologies must be considered during the differential diagnosis. These include hernias (abdominal wall, inguinal as well as femoral hernia [36], or especially in the case of women, the rare obturator hernia [37] ( $\bullet$ Fig. 7). Although genuine hernias are rare among athletes with groin pain ( $n=2 / 102$ patients [18]), MRI sequences with large field of view should nevertheless be checked for hernia, e.g. during a hip MRI [5]. Such hernias can be ascertained using the same sensitivity using ultrasound (always in comparison to the opposite side) as well as MRI [38]; however, ultrasonography permits dynamic examination at a higher time resolution.

A sports hernia is usually not an inguinal hernia, and the term "sports hernia" is not uniformly applied in the literature and is subject to debate $[1,5,17,18]$. The most frequent injuries clinically designated as sports hernia are musculoskeletal in nature and affect the pubic symphysis and the insertions of the muscles and tendons (see above); these are easily diagnosed using MRI [5, 17]. Other authors define a weak groin or sports hernia, which generally exhibits pain radiating into the genital region during coughing and sneezing, as a disruption of the rear wall of the inguinal canal, leading to nerve inflammation (genital branch of the genitofemoral nerve) and pain in the region of the tendon insertion on the pubic bone $[1,6,39]$. The fascia transversalis expands at its weakest point, thus resulting in a widening of the inguinal triangle. This consists of the rectus abdominis muscle, inguinal canal and epigastric vessels with consecutive cranial and medial displacement of the rectus abdominis muscle and increased tension on the pubic bone [39]. Dynamic ultrasonography can ascertain a protrusion of the rear abdominal wall (transverse fascia) under pressure (Valsalva maneuver) $[1,6]$, whereas a static MRI will be unremarkable. Among soccer players, extreme rotational and flexion motions in this region will provide above-average disclosure of a weak groin. This explanation is supported by an $80-90 \%$ success rate of microsurgery providing stabilization by overlapping layers of the transverse fascia [1]. Absence of pain has been reported after 14 days on average [39]. We draw the following conclusion: sports hernia is a clinical diagnosis $[3,6,28,38]$; the radiologist should employ MRI to exclude other causes or define for instance osteitis pubis as a correlate of a diagnosis of sports hernia.

\section{Labral Pathology of the Hip Joint}

The above-mentioned causes of groin pain must be distinguished from pain arising from other sources being projected into the groin. The most frequent cause is acetabular lab- 

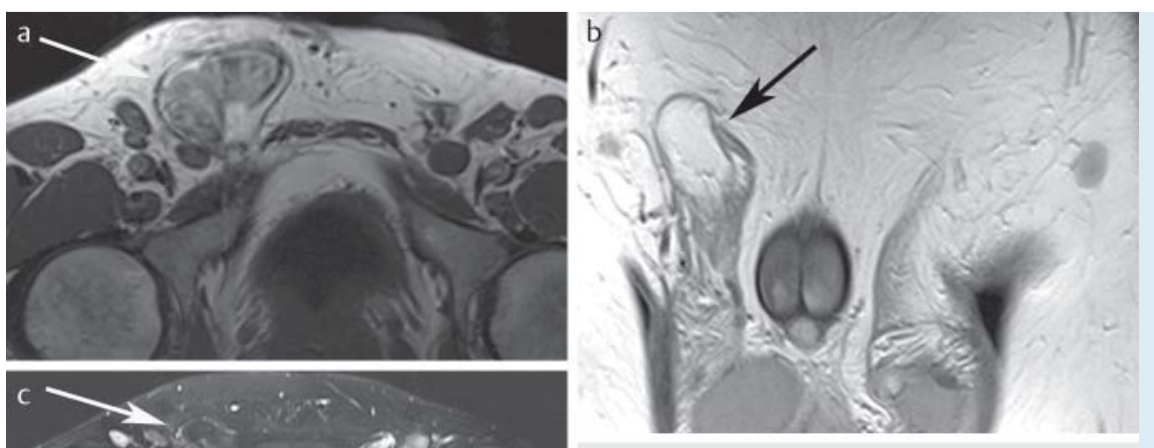

Fig. 7 Unsuspected hernia in MRI. The axial a and coronal b T1-weighted sequence of this 40-year-old athletic male with uncharacteristic groin pain demonstrates inguinal hernia (arrows). There is no fluid in the axial fat-suppressed T2-weighting around the herniated intestinal loop as an indicator of incarceration c. The latter image illustrates the value of sequences without fat suppression, since solely using fat-suppressed images makes it difficult

to delineate the hernias.

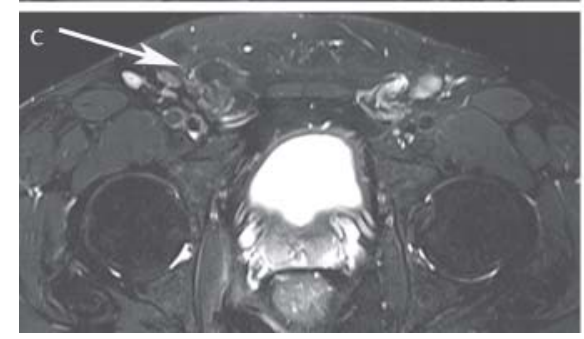

ral injury (in up to $22 \%$ of professional athletes with groin pain [40]) manifesting as pain radiating into the groin during hip movements [5, 17], particularly during rotational motions in the hip. Labral lesions are fostered by athletic actions (repetitive microtrauma) or isolated trauma during sports and femoroacetabular impingement arising from a poorly formed junction of the femoral head and acetabulum [40]. In the hip there is a distinction between pincer impingement when the femoral head is covered by a defective overhang of the acetabulum, and cam impingement, when the form-fit of the femoral head/neck juncture is reduced [41-43]. Most frequently exhibited (70\%) are combined forms and the antero-superior form of impingement with pain provocation, in particular during hip flexion and forced inner rotation and adduction [40, 41]. A labral tear can only be reliably diagnosed using direct MR arthrography ( $\bullet$ Fig. 8) [40, 44-47], although dedicated radially-arranged MR slices of the femoral head allow a more exact spatial relationship, since the labrum and cartilage are largely represented orthogonally [41]. Likewise, direct MR arthrography can distinguish between a labral tear and the more common detachment of the labrum caused by joint distension [45, 46]. MR arthrography achieves sensitivities/specificities of $90-95 \% /$ $91-100 \%$ compared to $30-65 \% / 36 \%$ when conventional MR imaging is used, since in the case of labral lesions, there is frequently no joint swelling leading to joint distension, which would make the tear more visible. In addition, a healthy labrum can exhibit heterogeneous signal intensity [40, 44, 46]. However, a normal labrum typically is triangular, and the signal intensity homogeneously ranges from none to low (Czerny stage 0 ) $[44,46]$. In cases of coagulation disorder or if the patient refuses joint puncture, indirect MR arthrography is an alternative to direct MR arthrography. The patient is brought to the MRI unit after administration of e.g. $0.2 \mathrm{ml} / \mathrm{kg}$ gadolinium-DTPA and 15 minutes of joint movement. Sensitivities of $88-100 \%$ have been described for labral tears. However, there was no improvement in the demonstration of cartilage compared to native MRI [48, 49]. CT arthrography is significantly inferior to MR arthrography in the detection of labral tears (MR sensitivity/specificity: $100 \% / 50 \%$ vs. CT: $15 \% / 13 \%$ ) [50]; therefore it should be used only in specific cases of MR contraindications and un- der consideration of weighing radiation exposure against potential information to be gained from patients who are generally young.

Imaging Protocol and Uncommon Differential Diagnoses Based upon the multiple above-described causes of groin pain, a two-stage MRI protocol ( $\bullet$ Table 3 ) can be used, consisting of overview images and targeted sequences [5]. Important in this regard are a coronal STIR sequence and an axial fat-suppressed T2-weighted turbo spin echo sequence of the entire pelvic region as search sequences which should demonstrate the anterior superior iliac spine, the ischial tuberosity, the pubic symphysis, the sacrum and the coccyx $[17,18]$. In addition, a non-fat suppressed, T1-weighted sequence to assess bone marrow should be integrated into the imaging protocol in order to diagnose myeloproliferative bone marrow changes or bone marrow metastasis, which, although unlikely, cannot be in general excluded as a cause of groin pain, due to increasing sports activity extending into old age. With respect to women, additional gynecologicalrelated pathologies such as endometriosis must be considered when making differential diagnoses [1, 17, 18]. Following these scan sequences with a large field of view, the protocol should be supplemented by targeted sequences with a small field of view, high resolution and slice thicknesses $\leq 3 \mathrm{~mm}$, e.g. to identify labral pathology or pubic symphysitis $[5,17]$.

\section{Summary and Critical Assessment \\ $\nabla$}

There are numerous differential diagnostic causes of groin pain in competitive athletes. Targeted MRI diagnostics play a key role in accurate diagnosis of, and therapy planning for, patients with recurring groin pain associated with sports. Close dialog between the radiologist and the sports medicine physician aids in concentrating the imaging procedure in order to rapidly provide the patient with a sufficiently focused radiological examination which can be assessed within a narrow clinical context. Since musculoskeletal injuries are frequently the source of groin pain among athletes, the MRI protocol should include imaging these regions in two planes, 

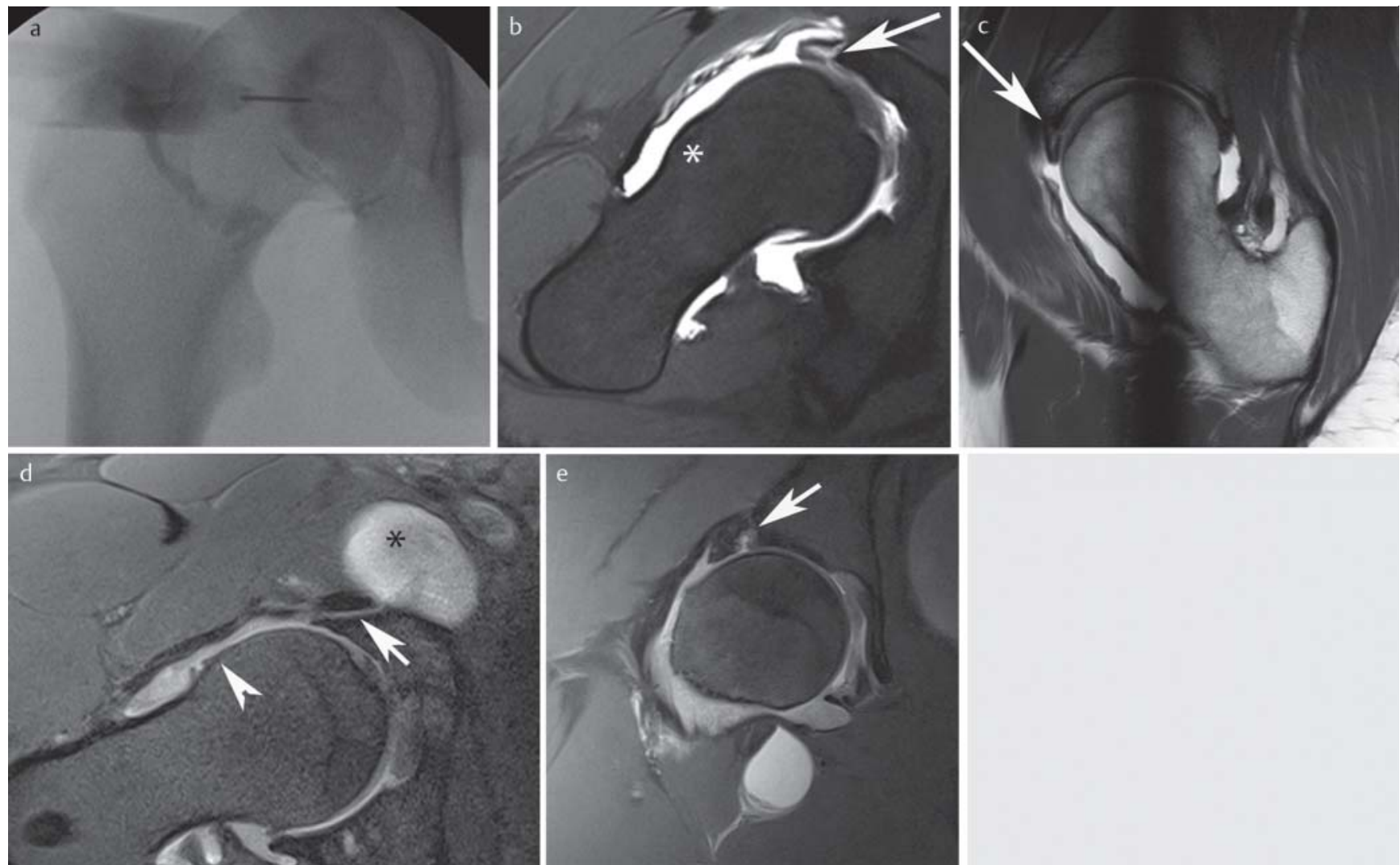

Fig. 8 Femoroacetabular impingement. a Joint puncture under fluoroscopy, test injection of $1-2 \mathrm{ml}$ iodine-based contrast medium to verify correct position, then injection of $10-20 \mathrm{ml}$ thinned gadolinium chelate, e. g. $0.0025 \mathrm{mmol} / \mathrm{ml}$ gadoteric acid. Pain provoked by joint distension is an indicator of labral lesion; likewise, pain reduction via intra-articular injection of e. g. $0.5 \%$ bupivacaine suggests that the source of the pain is in the joint $[45,46]$. MR arthrogram with axial fat-suppressed T1-weighting $\mathbf{b}$ and radial proton density (PD)-weighting c of a 22-year-old athletic woman with cam-type impingement due to an epi/metaphyseal bump (asterisk) and mucoid degenerative labral lesion (arrows). The radial MR sequence precisely illustrates the detachment and degeneration of the labrum. $\mathbf{d}$ MR arthrogram of 36-year-old athletic male with T1-weighted fat-suppressed axial sequence indicating iliopsoas bursitis (asterisk) with communication (arrow) between the iliopsoas bursa and joint (with $10-15 \%$ population [46]). The arrow tip points to the epi/metaphyseal bump with cam-type impingement ( $\alpha$-angle according to Nötzli: $75^{\circ}$, normal $\leq 55^{\circ}[42]$ ). e Demonstration of the labral tear in the coronal PD-weighted fatsuppressed sequence (arrow). whereby knowledge of specific localization and injury patterns will assist in diagnosing such pain reliably.

Finally it should be noted that although adductor problems and osteitis pubis are the most frequent reasons for interrupting training, in most cases these should be considered symptoms and not the cause of the injury. Therefore, in addition to these entities with a pathoanatomical/morphological correlate, the large group of muscular balance disturbances, impaired motions in muscle strings and blockages of various joints must be recognized as a source of groin pain. Radiologists thus have the important task of excluding morphologically identifiable causes described above when forming a diagnosis.

\section{Affiliations}

Department of Diagnostic and Interventional Radiology, University Hospital Heidelberg, Heidelberg/Germany

2 Sports Orthopaedics, Department of Orthopaedics, Trauma Surgery and Spinal Cord Injury Center, University Hospital Heidelberg, Heidelberg/Germany

3 Team physician of the German soccer league team TSG 1899 Hoffenheim

${ }^{4}$ Team physician of Germany national volleyball team and head of Sports Orthopaedics and Rehabilitation, Olympic Training Center Rhein-Neckar

\section{Acknowledgements:}

The authors dedicate this article to Prof. Dr. Hans-Ulrich Kauczor, Heidelberg, for his $50^{\text {th }}$ anniversary.

\section{References}

1 Best $R$, Nieß A, Striegel $H$. Die „Weiche Leiste“ als Differentialdiagnose chronischer Leistenbeschwerden beim Sportler. Dtsch Z Sportmed 2010; 61: 33-39

2 Cunningham PM, Brennan D, O'Connell $M$ et al. Patterns of bone and soft-tissue injury at the symphysis pubis in soccer players: observations at MRI. Am J Roentgenol 2007; 188: W291 - W296

3 MacMahon PJ, Hogan BA, Shelly MJ et al. Imaging of groin pain. Magn Reson Imaging Clin N Am 2009; 17: 655-666

4 Nielsen $A B$, Yde J. Epidemiology and traumatology of injuries in soccer. Am J Sports Med 1989; 17: 803-807

5 Omar IM, Zoga AC, Kavanagh EC et al. Athletic pubalgia and "sports hernia": optimal MR imaging technique and findings. Radiographics 2008; $28: 1415-1438$

6 Davies AG, Clarke AW, Gilmore J et al. Review: imaging of groin pain in the athlete. Skeletal Radiol 2010; 39: 629-644

7 Schneider S, Seither B, Tönges $S$ et al. Sports injuries: population based representative data on incidence, diagnosis, sequelae, and high risk groups. Br J Sports Med 2006; 40: 334-339 
8 Brown RA, Mascia A, Kinnear DG et al. An 18-year review of sports groin injuries in the elite hockey player: clinical presentation, new diagnostic imaging, treatment, and results. Clin J Sport Med 2008; 18: 221-226

9 Gabbe BJ, Bailey M, CookJL et al. The association between hip and groin injuries in the elite junior football years and injuries sustained during elite senior competition. Br J Sports Med 2010; 44: 799-802

10 Werner J, Hägglund $M$, Waldén $M$ et al. UEFA injury study: a prospective study of hip and groin injuries in professional football over seven consecutive seasons. Br J Sports Med 2009; 43: 1036-1340

11 LeBlanc KE, LeBlanc KA. Groin pain in athletes. Hernia 2003; 7: 68-71

12 Falvey EC, Franklyn-Miller A, MCCrory PR. The groin triangle: a pathoanatomical approach to the diagnosis of chronic groin pain in athletes. Br J Sports Med 2009; 43: 213-220

13 Renström P, Peterson L. Groin injuries in athletes. Br J Sports Med 1980; 14: $30-36$

14 Swan KG, Wolcott M. The athletic hernia: a systematic review. Clin Orthop Relat Res 2007; 455: 78-87

15 Anderson K, Strickland SM, Warren R. Hip and groin injuries in athletes. Am J Sports Med 2001; 29: 521-533

16 Mattes T, Fraitzl C, Ostertag $O$ et al. Differentialdiagnosen der aseptischen Hüftkopfnekrose - Artikulärer Leistenschmerz des Erwachsenen. Orthopäde 2007; 36: 414-422

17 Mullens FE, Zoga AC, Morrison WB et al. Review of MRI technique and imaging findings in athletic pubalgia and the "sports hernia". Eur J Radiol 2012; 81: 3780-3792

18 Zoga AC, Kavanagh EC, Omar IM et al. Athletic pubalgia and the "sports hernia": MR imaging findings. Radiology 2008; 247: 797-807

19 Rybak $L D$, Torriani M. Magnetic resonance imaging of sports-related muscle injuries. Top Magn Reson Imaging 2003; 14: 209-219

20 Fleckenstein JL, Weatherall PT, Parkey RW et al. Sports-related muscle injuries: evaluation with MR imaging. Radiology 1989; 172: 793 - 798

21 Connell DA, Schneider-Kolsky ME, Hoving JL et al. Longitudinal study comparing sonographic and MRI assessments of acute and healing hamstring injuries. Am J Roentgenol 2004; 183: 975-984

22 Slavotinek JP. Muscle injury: the role of imaging in prognostic assignment and monitoring of muscle repair. Semin Musculoskelet Radiol 2010; 14: 194-200

23 Müller-Wohlfahrt HW, Ueblacker P, Hänsel L (eds.) Muskelverletzungen im Sport. Stuttgart: Thieme; 2010, $200-207$

24 Peetrons $P$. Ultrasound of muscles. Eur Radiol 2002; 12: 35-43

25 Zarins B, Ciullo JV. Acute muscle and tendon injuries in athletes. Clin Sports Med 1983; 2: 167-182

26 Mueller-Wohlfahrt HW, Haensel L, Mithoefer K et al. Terminology and classification of muscle injuries in sport: The Munich consensus statement. Br J Sports Med 2013; 47: 342 - 350

27 Beatty T. Osteitis pubis in athletes. Curr Sports Med Rep 2012; 11: $96-98$

28 Schilders E, Talbot JC, Robinson P et al. Adductor-related groin pain in recreational athletes: role of the adductor enthesis, magnetic resonance imaging, and entheseal pubic cleft injections. J Bone Joint Surg Am 2009; 91: 2455-2460

29 Mishra A, Harmon K, Woodall J et al. Sports medicine applications of platelet rich plasma. Curr Pharm Biotechnol 2012; 13: 1185-1195

30 Barata $P$, Cervaens $M$, Resende $R$ et al. Hyperbaric oxygen effects on sports injuries. Ther Adv Musculoskel Dis 2011; 3: 111-121
31 Brennan D, O'Connell MJ, Ryan M et al. Secondary cleft sign as a marker of injury in athletes with groin pain: MR image appearance and interpretation. Radiology 2005; 235: 162 - 167

32 Koulouris $G$. Imaging review of groin pain in elite athletes: an anatomic approach to imaging findings. Am J Roentgenol 2008; 191: 962 -972

33 Robinson $P$, Bhat $V$, English $B$. Imaging in the assessment and management of athletic pubalgia. Semin Musculoskelet Radiol 2011; 15: 14-26

34 Robinson P, Salehi F, Grainger A et al. Cadaveric and MRI study of the musculotendinous contributions to the capsule of the symphysis pubis. Am J Roentgenol Am J Roentgenol 2007; 188: W440-W445

35 Shortt CP, Zoga AC, Kavanagh EC et al. Anatomy, pathology, and MRI findings in the sports hernia. Semin Musculoskelet Radiol 2008; 12 : $54-61$

36 Zandieh S, Nader A, Haller J. Die Hydrocele der Frau als Gimbernatische Hernie. Fortschr Röntgenstr 2011; 183: 855-857

37 Thiele J, Vothel F, Scheibe J. Hernia obturatoria - ein seltener Eingeweidebruch. Fortschr Röntgenstr 2011; 183: 393 -394

38 van den Berg JC, de Valois JC, Go PM et al. Detection of groin hernia with physical examination, ultrasound, and MRI compared with laparoscopic findings. Invest Radiol 1999; 34: 739-743

39 Minnich JM, Hanks J, Muschaweck U et al. Sports Hernia: diagnosis and treatment highlighting a minimal repair surgical technique. Am J Sports Med 2011; 39: 1341 - 1349

40 Groh MM, Herrera J. A comprehensive review of hip labral tears. Curr Rev Musculoskelet Med 2009; 2: 105-117

41 Fraitzl CR, Kappe T, Reichel H. Das femoroacetabuläre Impingement eine häufige Ursache des Leistenschmerzes beim Sportler. Dtsch Z Sportmed 2010; 61: 292-298

42 Potter HG, Schachar BA. High resolution noncontrast MRI of the hip. J Magn Reson Imaging 2010; 31: 268- 278

43 Ipach I, Mittag F, Sachsenmaier S et al. A new classification for "pistol grip deformity" - correlation between the severity of the deformity and the grade of osteoarthritis of the hip. Fortschr Röntgenstr 2011; 183: $365-371$

44 Czerny C, Oschatz E, Neuhold A et al. MR-Arthrographie des Hüftgelenks. Radiologe 2002; 42: 451 - 456

45 Petersilge CA. From the RSNA Refresher Courses. Radiological Society of North America. Chronic adult hip pain: MR arthrography of the hip. Radiographics 2000; 20: S43-S52

46 Petersilge CA. MR arthrography for evaluation of the acetabular labrum. Skeletal Radiol 2001; 30: 423-430

47 Fischer W, Bohndorf K, Kreitner KF et al. Indikationen der MR- und CTArthrografie - Empfehlungen der AG Muskuloskelettale Radiologie der DRG. Fortschr Röntgenstr 2009; 181: 441 - 446

48 Pozzi G, Stradiotti P, Parra CG et al. Femoro-acetabular impingement: can indirect MR arthrography be considered a valid method to detect endoarticular damage? A preliminary study. Hip Int 2009; 19: 386 391

49 Zlatkin MB, Pevsner D, Sanders TG et al. Acetabular labral tears and cartilage lesions of the hip: indirect MR arthrographic correlation with arthroscopy - a preliminary study. Am J Roentgenol 2010; 194: 709714

50 Perdikakis E, Karachalios T, Katonis $P$ et al. Comparison of MR-arthrography and MDCT-arthrography for detection of labral and articular cartilage hip pathology. Skeletal Radiol 2011; 40: 1441-1447 